\title{
Resonant inelastic x-ray scattering in one-dimensional copper oxides
}

\author{
K. Tsutsui, T. Tohyama, and S. Maekawa \\ Institute for Materials Research, Tohoku University, Sendai 980-8577, Japan
}

(Received 23 September 1999)

\begin{abstract}
The $\mathrm{Cu} K$-edge resonant inelastic x-ray scattering (RIXS) spectrum in one-dimensional insulating cuprates is theoretically examined by using the exact diagonalization technique for the extended one-dimensional Hubbard model with nearest-neighbor Coulomb interaction. We find the following characteristic features that can be detectable by RIXS experiments: (i) The spectrum with large momentum transfer indicates the formation of excitons, i.e. bound states of holon and doublon. (ii) The spectrum with small momentum transfer depends on the incident photon energy. We propose that the RIXS provides a unique opportunity to study the upper Hubbard band in one-dimensional cuprates.

PACS numbers: 78.20.Bh, 78.70.Ck, 78.66.Nk, 71.10.Fd
\end{abstract}

Insulating copper oxides, when mapped onto a singleband component, 1 have their occupied lower Hubbard band (LHB) and the unoccupied upper Hubbard band (UHB) separated by a Mott gap. Angle-resolved photoemission spectroscopy (ARPES) has succeeded in observing the momentum dependence of the LHB for twodimensional (2D) (Ref. 2) and 1D (Ref. 3) cuprates. In contrast to the LHB, the UHB is far from our understanding.

The information about the UHB can be extracted from excitations from the LHB to UHB across the Mott gap. A representative technique for this is electron energy-loss spectroscopy (EELS), which has been applied to both 2D (Ref. 4) and 1D (Ref. 5) insulating cuprates. Another technique is resonant inelastic x-ray scattering (RIXS). When the incident photon energy $\omega_{i}$ is tuned through the $\mathrm{Cu} K$ absorption edge, the momentum dependence of the excitations from the LHB to UHB has been observed for insulating 2D cuprates, $\mathrm{Sr}_{2} \mathrm{CuO}_{2} \mathrm{Cl}_{2}$ (Ref. 6) and $\mathrm{Ca}_{2} \mathrm{CuO}_{2} \mathrm{Cl}_{2}$ (Ref. 7). On the theoretical side, the present authors predicted the dependence based on a $2 \mathrm{D}$ Hubbard model with second- and third-neighbor hopping terms.

Typical examples of $1 \mathrm{D}$ cuprates are $\mathrm{Sr}_{2} \mathrm{CuO}_{3}$ and $\mathrm{SrCuO}_{2}$. Their magnetic susceptibilities are explained by the 1 D Heisenberg model. 9 ARPES measurement 3 , 10 showed that a photodoped hole cannot exist as a quasiparticle, but changes into two collective excitations, a spinon and a holon, as expected from the 1D single-band Hubbard and $t-J$ models. From EELS experiments, it has been pointed out that an extended version of the Hubbard model with a moderate nearest-neighbor Coulomb interaction $V$ is necessary to understand excitations across the Mott gap. It is interesting to know what we obtain in the RIXS spectrum prior to the experiments.

In this study, the momentum dependence of the RIXS spectrum in 1D cuprates is demonstrated by using the exact diagonalization technique for the extended Hubbard model. We find the following characteristic features that can be detectable by RIXS experiments: The spectral weight concentrates on the narrow energy region due to the formation of excitons. This is also seen in the dynamical charge-correlation function, although the dependence of the intensity on the momentum transfer is different. We also find that the spectral weight distribution for a small momentum transfer depends on the incident photon energy. This is associated with the intermediate state that is characteristic of the RIXS process.

A minimal model that can describe the LHB and UHB is the extended Hubhard model as used in the analyses of the EELS spectra. 3 Hereafter, we use the term " $3 d-$ electron system" to stand for the LHB and UHB. The Hamiltonian is given by

$$
\begin{aligned}
H_{3 d}= & -t \sum_{i, \sigma}\left(d_{i, \sigma}^{\dagger} d_{i+1, \sigma}+\text { H.c. }\right)+U \sum_{i} n_{i, \uparrow}^{d} n_{i, \downarrow}^{d} \\
& +V \sum_{i} n_{i}^{d} n_{i+1}^{d},
\end{aligned}
$$

where $d_{i, \sigma}^{\dagger}$ is the creation operator of $3 d$ electron with spin $\sigma$ at site $i, n_{i, \sigma}^{d}=d_{i, \sigma}^{\dagger} d_{i, \sigma}$, and $n_{i}^{d}=n_{i, \uparrow}^{d}+n_{i, \downarrow}^{d}$. The onsite Coulomb energy $U$ corresponds to the charge-transfer energy of cuprates.

In the intermediate states of the $\mathrm{Cu} K$-edge RIXS process, $3 d$ electrons interact with a $1 s$-core hole created by the dipole transition of an $1 s$ electron to $4 p$ orbital due to absorption of an incident photon with energy $\omega_{i}$ and momentum $\mathbf{K}_{i}$. This interaction is written as

$$
H_{1 s-3 d}=-V_{c} \sum_{i, \sigma, \sigma^{\prime}} n_{i, \sigma}^{d} n_{i, \sigma^{\prime}}^{s}
$$

where $n_{i, \sigma}^{s}$ is the number operator of the $1 s$-core hole with spin $\sigma$ at site $i$, and $V_{c}$ is taken to be positive. This interaction $V_{c}$ causes excitations of $3 d$ electrons across the gap. The photoexcited $4 p$ electron is assumed to go into the bottom of the $4 p$ band with momentum $\mathbf{k}_{0}$ and not to interact with either the $3 d$ electrons or $1 s$-core hole due to the delocalized nature of the $4 p$ orbital. In the final state, the $4 p$ electron goes back to the $1 s$ orbital, emitting a photon with energy $\omega_{f}$ afd momentum $\mathbf{K}_{f}$. The RIXS spectrum is then given by 


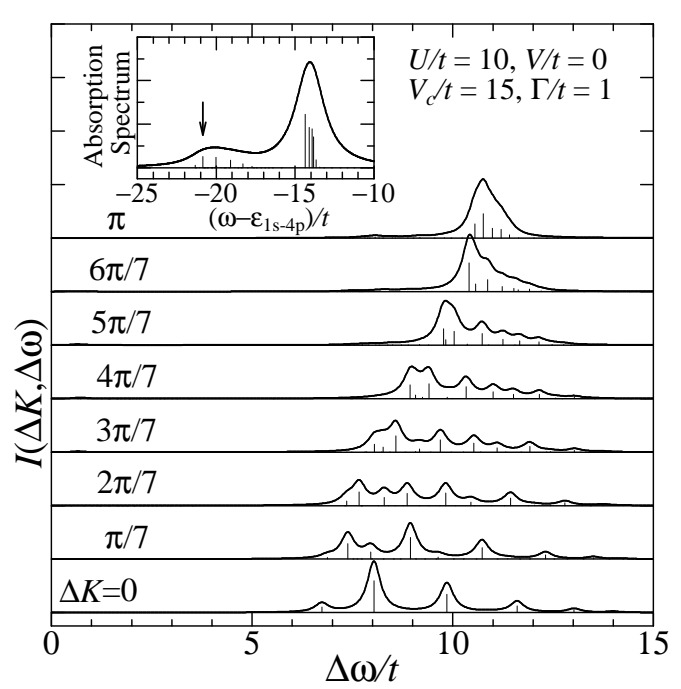

FIG. 1. Resonant inelastic x-ray scattering spectra for $\mathrm{Cu}$ $K$-edge in the 1D half-filled Hubbard model. The spectra of the elastic scattering process at $\Delta K=0$ are not shown. The parameters used are $U / t=10, V / t=0, V_{c} / t=15$, and $\Gamma / t=1$. The $\delta$ functions (the vertical thin solid lines) are convoluted with a Lorentzian broadening of $0.2 t$. The inset is the $\mathrm{Cu} 1 s$ absorption spectra with a broadening of $1.0 t$, and the incident photon energy $\omega_{i}$ is set to the value denoted by the arrow.

$$
\begin{aligned}
& I(\Delta \mathbf{K}, \Delta \omega)=\sum_{\alpha} \mid\langle\alpha| \sum_{\sigma} s_{\mathbf{k}_{0}-\mathbf{K}_{f}, \sigma} p_{\mathbf{k}_{0}, \sigma} \\
& \quad \times\left.\frac{1}{H+\varepsilon_{1 s-4 p}-E_{0}-\omega_{i}-i \Gamma} p_{\mathbf{k}_{0}, \sigma}^{\dagger} s_{\mathbf{k}_{0}-\mathbf{K}_{i}, \sigma}^{\dagger}|0\rangle\right|^{2} \\
& \quad \times \delta\left(\Delta \omega-E_{\alpha}+E_{0}\right),
\end{aligned}
$$

where $H=H_{3 d}+H_{1 s-3 d}, \Delta \mathbf{K}=\mathbf{K}_{i}-\mathbf{K}_{f}, \Delta \omega=\omega_{i}-\omega_{f}, s_{\mathbf{k}, \sigma}^{\dagger}$ $\left(p_{\mathbf{k}, \sigma}^{\dagger}\right)$ is the creation operator of the $1 s$-core hole ( $4 p$ electron) with momentum $\mathbf{k}$ and spin $\sigma,|0\rangle$ is the ground state of the half-filled system with energy $E_{0},|\alpha\rangle$ is the final state of the RIXS process with energy $E_{\alpha}, \Gamma$ is the inverse of the relaxation time in the intermediate state, and $\varepsilon_{1 s-4 p}$ is the energy difference between the $1 s$ level and the bottom of the $4 p_{z}$ band. The momentum component parallel to the $1 \mathrm{D} \mathrm{Cu}$ chain is represented by $\Delta K$ hereafter. The values of the parameters are set to be $U / t=10, V_{c} / t=15$, and $\Gamma / t=1$ as for the $2 \mathrm{D}$ cuprates. The RIXS spectrum in Eq. (3) is calculated on a 14-site ring by using a modified version of the conjugate-gradient method together with the Lanczös technique.

Figure 11 shows the momentum dependence of the RIXS spectrum for the case of $V / t=0$. The inset shows the ab sorption spectrum of the $1 s$ electron to the $4 p$ orbital. The incident photon energy $\omega_{i}$ that appears in Eq. (3) is set to be the value denoted by the arrow. This means that the states near the arrow satisfy the resonance conditions of Eq. (3). For small $\Delta K$, the spectra spread over about $8 t$. The energy region, however, shrinks with increasing $\Delta K$, and the spectral weight concentrates on a narrow

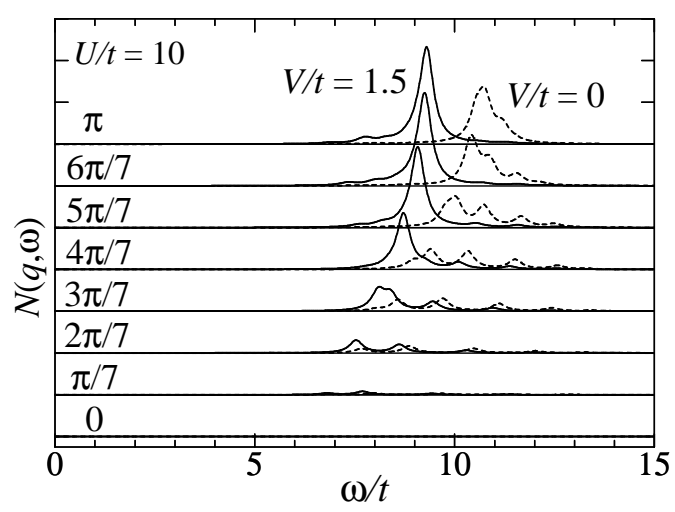

FIG. 2. Dynamical charge correlation function $N(q, \omega)$ in the 1D half-filled Hubbard model. The parameters used are $U / t=10, V / t=0$ (broken lines), and $V / t=1.5$ (solid lines). The $\delta$-functions are convoluted with Lorentzian broadening of $0.2 t$.

energy region at $\Delta K=\pi$. This momentum dependence is similar to that of the dynamical charge-correlation function $N(q, \omega)$, shown by dashed lines in Fig. 2, which is explained by the particle-hole model with only a charge degree of freedom. 511 In the particle-hole model, holon and doublon bands separated by $U$ describe the LHB and UHB, respectively. We note that the spin degree of freedom is less effective on $N(q, \omega)$ because of the wavefunction factorization 12 in the large- $U$ limit. At the zone center of the interband transitions, one obtains a continuum with a width of $8 t$ above the gap of $U-4 t$. The width of the continuum decreases with an increase of the momentum transfer, and finally becomes an excitation with energy $U$ at the zone boundary. This momentum dependence is qualitatively consistent with that shown in Fig. 11 and also with $N(q, \omega)$ shown in Fig. 2. It is interesting to note that the integrated weight of $I(\Delta K, \Delta \omega)$, with respect to $\Delta \omega$, is almost independent of $\Delta K$ in contrast to $N(q, \omega)$ where the integrated ight is proportional to $\sin ^{2}(q / 2)$ for the large- $U$ region 11

The effect of the nearest-neighbor Coulomb interaction $V$ on $I(\Delta K, \Delta \omega)$ is shown in Fig. 3, where $V / t \overline{-1.5}$ is taken according to the analyses of the EELS data. 3 From the comparison between Figs. 1 and 3 , we find that the shape of the spectrum changes remarkably for $\Delta K>\pi / 2$, accompanied by the formation of sharp peaks together with the shift of the spectral weight to the lower-energy region. This is due to excitons that appear at the momenta satisfying a condition that $V>2 t \cos (\Delta K / 2) .11 \mathrm{~A}$ similar change is also seen in $N(q, \omega)$ (see Fig.2). For $\Delta K<\pi / 2$, the intensity of the spectrum near the lower edge is enhanced by the presence of excitonic effects, which is again similar to the feature of $N(q, \omega) .11$ We note that the presence of $V$ also affects the RIXS spectrum in the $2 \mathrm{D}$ case, although the edge structure, which is a fingerprint of the shape of the UHB, 8 does not depend on $V$.

The spectral weight depends on the incident photon en- 


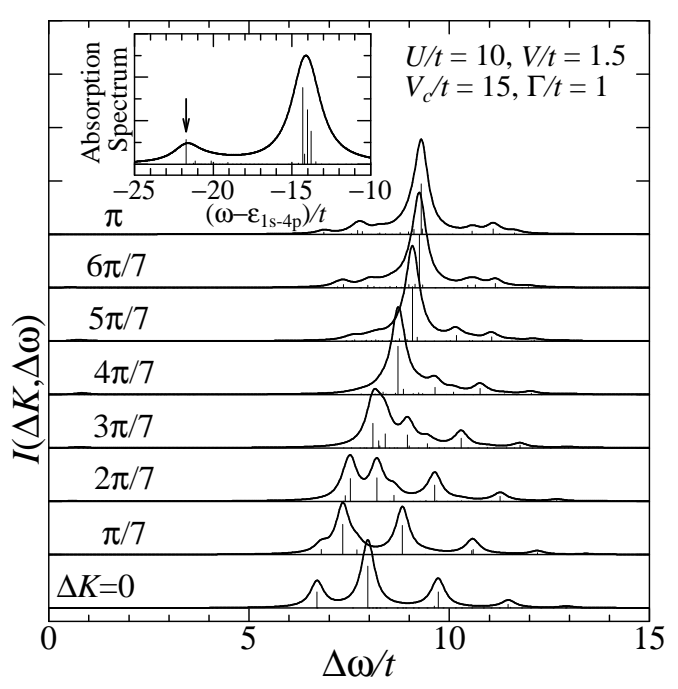

FIG. 3. The same as Fig. 1 but for $V / t=1.5$.

ergy $\omega_{i}$. By changing $\omega_{i}-\varepsilon_{1 s-4 p}$ from about $-21 t$ to $-14 t$, the distribution of intensity in the lower-momentum region shifts to the higher-energy region, as shown in Fig. 4. This can be attributed to the nature of the intermediate state around the resonance condition, denoted by the arrows in the insets of Figs. 11 and 3. The peaks at $\omega-\varepsilon_{1 s-4 p} \sim-21 t\left(\sim U-2 V_{c}\right)$ in the absorption spectrum are characterized by states having configurations with the core-hole site doubly occupied by $3 d$ electrons together with an empty site of $3 d$ electrons. On the other hand, in states near $\omega-\varepsilon_{1 s-4 p}=-14 t\left(\sim-V_{c}\right)$, the corehole site is singly occupied by $3 d$ electrons and there is no empty site. Since the different intermediate states are used depending on the incident photon energy, the RIXS spectrum depends on the energy. The spectrum in the low-energy region of $\Delta \omega / t \lesssim 1$ in Fig. 目 corresponds to singlet excitations of the spinon pair, because the intermediate state has singly occupied configurations.

In summary, we have examined the momentum dependence of the RIXS spectrum in 1D cuprates, which provides a unique opportunity to study the upper Hubbard band, using the exact diagonalization technique for the extended Hubbard model. We have found the following characteristic features of the RIXS spectrum: The spectrum with large momentum transfer, $\Delta K>\pi / 2$, indicates the formation of excitons, i.e., bound states of a holon and a doublon. We have also found that the spectral weight distribution for small momentum transfer, $\Delta K<\pi / 2$, depends on the incident photon energy. The dependence is associated with the intermediate state that is characteristic of the RIXS process. These findings will be good guides for RIXS experiments on 1D insulating cuprates that will be performed in the future.

This work was supported by Priority-Area Grants from the Ministry of Education, Science, Culture and Sport of Japan, CREST, and NEDO. Computations were carried out in ISSP, University of Tokyo; IMR,Tohoku Univer-

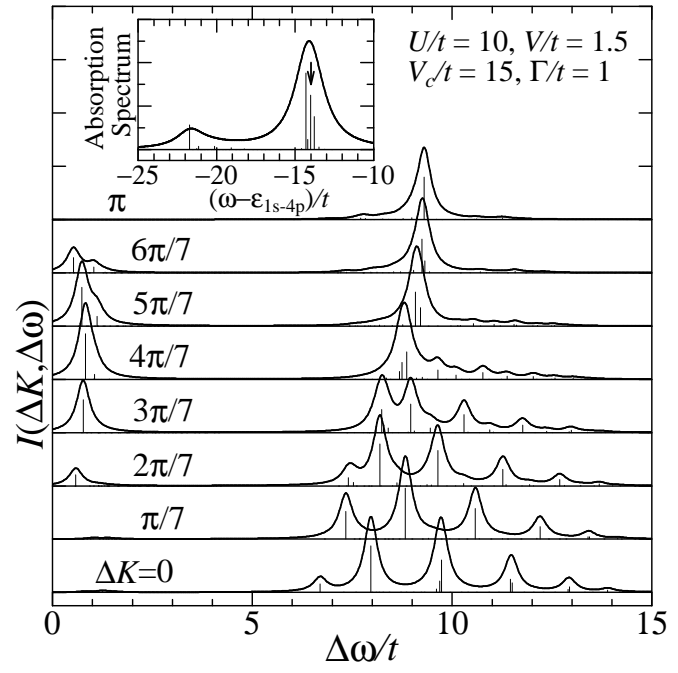

FIG. 4. The same as Fig. 3, but with the incident photon energy $\omega_{i}$ set to the value denoted by the arrow in the inset.

sity; and Tohoku University.

${ }^{1}$ F. C. Zhang and T. M. Rice, Phys. Rev. B 37, 3759 (1988).

${ }^{2}$ B. O. Wells, Z.-X. Shen, A. Matsuura, D. M. King, M. A. Kastner, M. Greven, and R. J. Birgeneau, Phys. Rev. Lett. 74, 964 (1995).

${ }^{3}$ C. Kim, A. Y. Matsuura, Z.-X. Shen, N. Motoyama, H. Eisaki, S. Uchida, T. Tohyama, and S. Maekawa, Phys. Rev. Lett. 77, 4054 (1996); C. Kim, Z.-X. Shen, N. Motoyama, H. Eisaki, S. Uchida, T. Tohyama, and S. Maekawa, Phys. Rev. B 56, 15589 (1997).

${ }^{4}$ Y. Y. Wang, F. C. Zhang, V. P. Dravid, K. K. Ng, M. V. Klein, S. E. Schnatterly, and L. L. Miller, Phys. Rev. Lett. 77, 1809 (1996).

${ }^{5}$ R. Neudert, M. Knupfer, M. S. Golden, J. Fink, W. Stephan, K. Penc, N. Motoyama, H. Eisaki, and S. Uchida, Phys. Rev. Lett. 81, 657 (1998).

${ }^{6}$ P. Abbamonte, C. A. Burns, E. D. Isaacs, P. M. Platzman, L. L. Miller, S. W. Cheong, and M. V. Klein, Phys. Rev. Lett. 83, 860 (1999).

${ }^{7}$ Z. Hasan, E. D. Issacs, Z.-X. Shen, L. L. Miller, K. Tsutsui, T. Tohyama, and S. Maekawa (unpublished).

${ }^{8}$ K. Tsutsui, T. Tohyama, and S. Maekawa, Phys. Rev. Lett. 83, 3705 (1999).

${ }^{9}$ N. Motoyama, H. Eisaki, and S. Uchida, Phys. Rev. Lett. 76, 3212 (1996).

${ }^{10}$ H. Fujisawa, T. Yokoya, T. Takahashi S. Miyasaka, M. Kibune, and H. Takagi, Phys. Rev. B 59, 7358 (1999).

${ }^{11}$ W. Stephan and K. Penc, Phys. Rev. B 54, R17269 (1996).

${ }^{12}$ F. Woynarovich, J. Phys. C 15, 97 (1982); M. Ogata and H. Shiba, Phys. Rev. B 41, 2326 (1990). 